\title{
Complete SAXS data analysis and synthesis of lamellar two-phase systems. Deduction of a simple model for the layer statistics
}

\section{N. STRIBECK}

Universität Hamburg, Inst. f. Techn. u. Makromol. Chemie, Bundesstr. 45, 20146 Hamburg 28, Germany

A structural model for the analysis of SAXS data from lamellar two-phase systems is proposed and applied on data sets from three injection moulded poly(ethylene terephthalate) (PET) samples. The concept of data analysis is based on Ruland's interface distribution function (IDF). The suggested model is defined by few parameters of physical meaning. It unifies the well known concepts of an ensemble of non-uniform stacks, finite stack height and one-dimensional paracrystalline disorder in an analytical expression. In order to deduce this expression, the notion of an inhomogeneous structure within the sample is mathematically treated in terms of "compansion", a general superposition principle. Its mathematical equivalent in one dimension is the Mellin convolution. The theory of the Mellin convolution may be used to find analytical functions even for the convoluted. An example is given, which in future work may be used to describe the thickness distributions of amorphous and crystalline layers. In the application part of this study gaussians are used to describe the thickness distributions in each local stack. The introduction of compansion adds one extra parameter, which describes the heterogeneity of the sample. Compansion makes the global thickness distributions become more asymmetrical.

\section{Introduction}

A frequently studied type of superstructure in polymers is the lamellar two-phase system. Small angle $x$-ray scattering is a common method of research in this field, but it affords appropriate evaluation methods and structure modelling in order to gain information on global parameters which characterize the structure. Even the restricted objective of only determining the "long period" from the reflection maxima is not a simple task, as Reinhold et. al. (1) have shown. In order to explain the curve, the authors suggest a special type of asymmetric function to describe the frequency distribution of long periods. Asymmetric distributions have several times been considered for two-phase systems (2-5). Other authors (6-8) conclude, that the ensemble of lamellar stacks shows inhomoge- neity in the sense, that the long period varies from stack to stack.

An advanced method for SAXS data evaluation is Ruland's method of "interface distribution function (IDF) analysis" (9). The IDF $g_{1}(r)$ is an image of the scattering intensity in physical space, which describes the structure of a lamellar systems by a series of distance distributions, $h_{i}\left(d_{i}, r\right)$, where $d_{i}$ is the corresponding average distance between two deliberate phase interfaces along the main axis of the representative lamellar stack.

\section{Theoretical}

If the structure of a sample is heterogeneous, i. e. the structure of the lamellar stacks varies from region to region, it may be difficult to extract a model structure (e.g. a 1Dparacrystal) from the scattering curve. 
Nevertheless to a first approximation an average reference structure $g_{1 r}(r)$ may exist, which after only a compression or expansion fits the local stacks. The author has named this principle "compansion" (10). Thus one introduces a frequency distribution $h_{H}(r)$ of compression/expansion factors and finds

$$
g_{1}(r)=\int_{0}^{\infty} h_{H}(y) g_{1 r}\left(\frac{r}{y}\right) \frac{d y}{y}:=h_{H}(r) \otimes g_{1 r}(r)
$$

for the observed structure $g_{1}(r)$. Eq. (1) describes the Mellin convolution $(11,12)$. With the definition of the "i-th moment about origin" of a distribution $h(r)$,

$$
\mu_{i, h}^{\prime}=\int_{0}^{\infty} r^{i} h(r) d r
$$

$h_{H}(r)$ enjoys the properties

$$
\mu_{0, h_{H}}^{\prime}=1, \quad \mu_{1, h_{H}}^{\prime}=1,
$$

i. e. norm and mean are equal to unity.

Since $g_{1 r}(r)$ can be expanded into a series of distance distributions $h_{i}(r)$, the problem is reducible to the study of the "Mellin convoluted" from functions satisfying eq. (3).

Consider gaussian distributions, $h(r, \sigma)$, which satisfy eq. (3)

$$
h(r, \sigma)=\frac{1}{\sqrt{2 \pi} \sigma} \exp \left(-\frac{(r-1)^{2}}{2 \sigma^{2}}\right)
$$

and let $f(r):=h_{H}\left(r, \sigma_{H}\right) \otimes h_{g}\left(r, \sigma_{g}\right)$ be the companded. Then $F(s)$, the Fourier transform of $f(r)$, can be given in analytical form (cf. (10))

$$
F(s)=\sqrt{A(s)} \exp (-A(s) B(s)) \exp (2 \pi i s A(s))
$$

with

$$
\mathrm{A}(\mathrm{s})=\frac{1}{1+\left(2 \pi \sigma_{g} \sigma_{H} s\right)^{2}}, B(s)=2 \pi^{2}\left(\sigma_{g}^{2}+\sigma_{H}^{2}\right) s^{2} \text {. }
$$

Examples for these functions are shown in Fig. 1. As one can see, a smooth transition from symmetrical to skew distributions is the natural result of increasing heterogeneity under compansion.
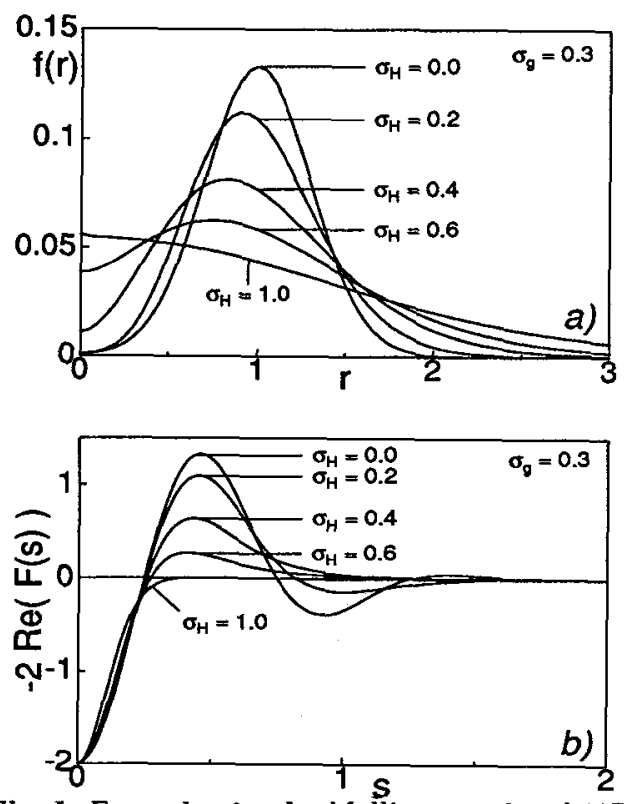

Fig. 1: Examples for the Mellin convoluted (IDcompanded) of two gaussians as a function of the width parameter $\sigma_{H}$ of one of the functions. a) the convoluted function $f(r)$ in physical space. b) $-2 \operatorname{Re}(F(s))$, the negative Fourier transform of $f(r)$. Such terms occur in $s^{4} I(s)$.

Gaussians and their companded do not vanish for $r \leq 0$. Following a method of Marichev (12), one may find numerous different basic distributions, which overcome this drawback. Moreover, their Mellin convoluted may be calculated analytically. A simple example is

$$
h(a, r)=\frac{a^{a}}{\Gamma(a)} r^{a-1} \exp (-a r), \quad a \geq 2 .
$$

Its $i$-th moment about origin, $\mu_{i, h}^{\prime}(a)$ is a function of the variable $a$

$$
\mu_{i, h}^{\prime}(a)=\frac{\Gamma(a+n)}{\Gamma(a)} \frac{1}{a^{n}}=\frac{(a)_{n}}{a^{n}} .
$$

$(a)_{n}$ is Pochhammer's function. The standard deviation of $h(a, r)$ is $\sigma^{2}=1 / a$, its skewness $\gamma_{1}=2 / \sqrt{a}$. With increasing $a$ the distribution becomes narrower and more symmetrical (cf. Fig. 2). 


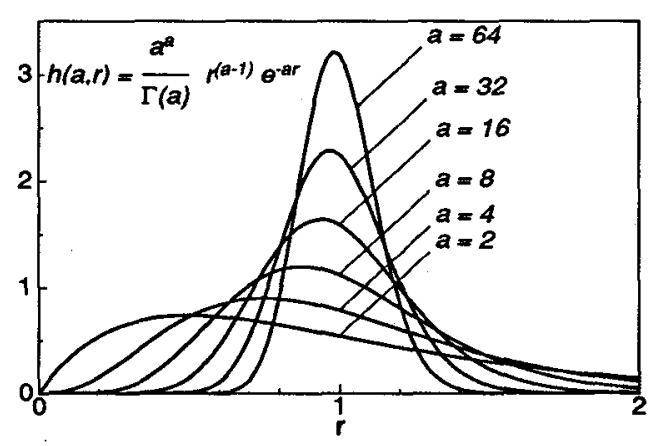

Fig. 2: Sketch of exemplary model distributions $h(a, r)$, which vanish for $r<0$ and the Mellin convoluted of which is analytical.

For $f(a, b, r)=h(a, r) \otimes h(b, r)$ one finds

$f(r)=\frac{2(a b)^{(a+b) / 2}}{\Gamma(a) \Gamma(b)} r^{(a+b-2) / 2} K_{a-b}(2 \sqrt{a b r})$

with $\mathrm{K}_{n}(x)$ being the modified Bessel function of the second kind and order $n$.

\section{Example evaluation and results}

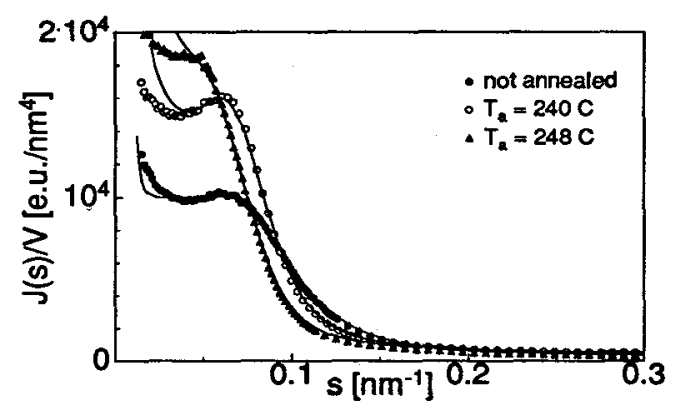

Fig. 3: Slit-smeared SAXS intensities $J(s) / V$ of three injection moulded PET samples. The solid lines show curves synthesized from the parameter values gained from complete data analysis.

The measured SAXS curves $J(s) / V$ from three injection moulded PET samples are shown in Fig. 3. Two of the samples have been annealed at different temperatures. The solid lines show the curves, which have been synthesized from the parameter values obtained in the model fits. The deviations at small $s$-values are discussed elsewhere (10).

The IDFs $g_{1}(r)$, which have been computed from $J(s) / V$, are shown in Fig. 4. For a detailed discussion of the evaluation steps cf. (10).

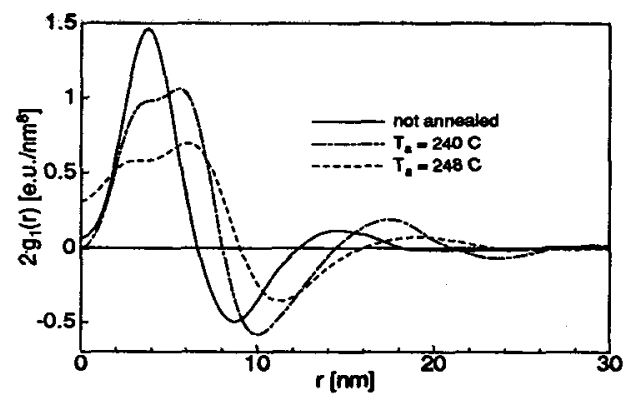

Fig. 4: Interface distributions $g_{1}(r)$ obtained from $J(s) / V$.

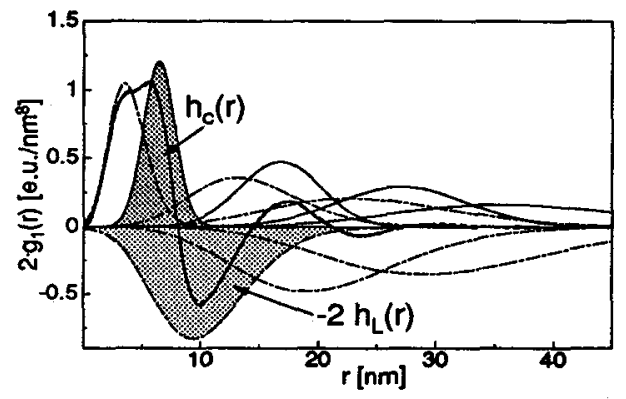

Fig 5: Model decomposition after fit of the sample annealed at $240 C$. Two global distance distributions are marked as $h_{c}(r)$ and $-2 h_{L}(r)$.

The model here assumes compansion but no specific statistical model (like paracrystal statistics).

The model decomposition in Fig. 5 shows the overlapping of the global distance distributions $h_{i}\left(d_{i}, \sigma_{i}, r\right) \otimes h_{H}\left(\sigma_{H}, r\right)$ and the asymmetry due to compansion in, e. g., the distribution of the long periods $-2 h_{L}(r)$. The symmetry of some special distributions (thin solid lines) led to the conclusion, that here the stacks have a finite height. So the drawing only represents one intermediate step in the model refining process. After considering compansion, the increase of the widths $\sigma_{i}$ with increasing distance does no longer contradict a paracrystalline statistics.

Only for the unannealed sample the stack height is high enough to allow for a fit under the assumption of an infinite stack height. 


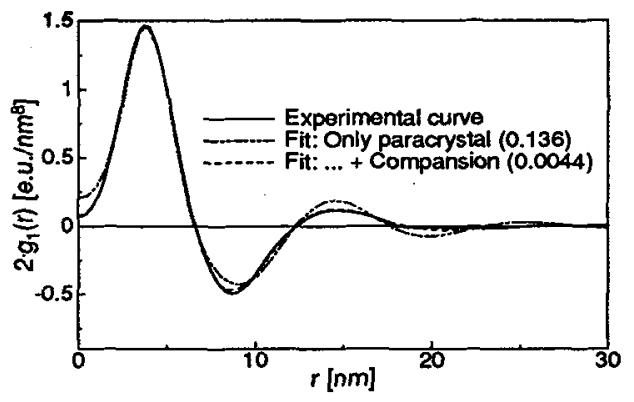

Fig. 6: Two model fits for the unannealed sample, assuming the statistical model of an infinite paracrystal stack. In brackets: the residual sum of squares of the fit.

Corresponding fits are shown in Fig. 6. One can see, that the assumption of compansion improves the fit considerably: Only by introduction of one additional model parameter, the residual sum of squares decreases by a factor of 31 .

Table 1: Parameters of the structural model for three PET samples under consideration of sample heterogeneity (compansion). $A_{P}$ is a weight parameter, $\sigma_{H}$ is the heterogeneity of the sample. $d_{c}$ and $d_{a}$ are the average thicknesses of crystalline and amorphous lamellae. $\sigma_{c} / d_{c}$ and $\sigma_{a} / d_{a}$ are the relative standard deviations of the reference stack. $n$ is the number of crystalline lamellae, which belong to a finite stack.

\begin{tabular}{|l|c|c|c|}
\hline$T_{a}[C]$ & - & $240 \mathrm{C}$ & $248 \mathrm{C}$ \\
\hline$A_{P}\left[e l / \mathrm{nm}^{8}\right]$ & 1.74 & 1.77 & 1.64 \\
\hline$\sigma_{H}$ & 0.21 & 0.24 & 0.24 \\
\hline$d_{c}[\mathrm{~nm}]$ & 4.3 & 6.5 & 7.2 \\
\hline$d_{a}[\mathrm{~nm}]$ & 4.7 & 4.0 & 3.9 \\
\hline$\sigma_{c} / d_{c}$ & 0.26 & 0.02 & 0.04 \\
\hline$\sigma_{a} / d_{a}$ & 0.41 & 0.40 & 0.66 \\
\hline$n$ & 10 & 5 & 4 \\
\hline
\end{tabular}

Finally, Table 1 shows the results of the analysis for all three samples. With increasing annealing the long period only slightly increases, but the crystallites grow and the correlation along the stack breaks down.

\section{Conclusions}

This study shows that the frequently discussed models for the statistics in an ensemble of lamellar stacks may be superimposed. Compansion considers this superposition to a first approximation and generates asymmetric frequency distributions in a natural manner. As has been shown in an example, the study of the properties of the Mellin convolution may yield interesting types of frequency distribution functions, which might be more suitable than gaussians for the analysis of SAXS from lamellar or fibrillar two-phase systems.

For all the samples studied here heterogeneity appears to be an essential feature of the structure, and thus has to be considered.

\section{References}

[1] Reinhold C, Fischer EW, Peterlin A (1964) J Appl Phys 35:71-74

[2] Strobl GR, Schneider MJ, Voigt-Martin IG (1980) J Polym Sci Phys B18:1361-1381

[3] Hall IH, Mahmoud EA, Carr PD, Geng YD (1987) Colloid Polym Sci 265:383-393

[4] Voigt-Martin IG, Mandelkern L (1989) J Polym Sci Phys B27:967-991

[5] Hanna S, Windle AH (1988) Polymer 29:207-223

[6] Porod G (1951) Colloid Polym Sci 124:83114

[7] Strobl GR, Müller N (1973) J Polym Sci Phys 11:1219-1233

[8] Stribeck N, Ruland W (1978) J Appl Cryst 11:535-539

[9] Ruland W (1977) Colloid Polym Sci 255:417-427

[10] Stribeck N (1993) Colloid Polym Sci, in press

[11] Titchmarsh EC (1948) Introduction to the Theory of Fourier Integrals, 2nd ed., Clarendon press, Oxford, p. 53

[12] Marichev OI (1983) Handbook of Integral Transforms of Higher Transcendental Functions: Theory and Algorithmic Tables, Wiley, New York 